\title{
A Case Report of Sweet's Syndrome with Parotitis
}

\author{
Myoung Soo Jo ${ }^{1}$, Young Bin $\operatorname{Lim}^{1}$, Hea Kyeong Shin ${ }^{1}$, Joon Choe ${ }^{1}$, Jung Hyun Seul ${ }^{1}$, \\ Tae Jung Jang ${ }^{2}$ \\ Departments of ${ }^{1}$ Plastic and Reconstructive Surgery, and ${ }^{2}$ Pathology, Dongguk University College of Medicine, Gyeongju, Korea
}

\begin{abstract}
Sweet's syndrome is characterized by clinical symptoms, physical features, and pathologic findings which include fever, neutrophilia, tender erythematous skin lesions, and a diffuse infiltrate of mature neutrophils. This is a report of our experience of Sweet's syndrome with parotitis. A 57-year-old man initially presented with tender swelling on the right cheek similar to parotitis. His symptoms relapsed despite the use of an oral antibiotic agent for 3 weeks. He additionally presented with erythematous papules and plaques on the periocular area and dorsum of both hands. Histiopathologic findings on punch biopsy of the right dorsum of the hand showed superficial perivenular histiocytic infiltration without vasculitis. We confirmed this as histiocytoid Sweet's syndrome and used systemic corticosteroid. After initiation of treatment with systemic corticosteroids, there was a prompt recovery from both the dermatosis-releated symptoms and skin lesions. Sweet's syndrome should be considered in patients with therapy-refractory parotitis and unclear infiltrated nodules. We present a confusing case who initially appeared to have parotitis but turned out to have histiocytoid Sweet's syndrome.
\end{abstract}

Keywords Sweet syndrome / Dermatosis / Parotitis

\author{
Correspondence: Jung Hyun Seul \\ Department of Plastic and \\ Reconstructive Surgery, Dongguk \\ University College of Medicine, \\ 87 Dongdae-ro, Gyeongju 780-350, \\ Korea \\ Tel: +82-54-770-8243 \\ Fax: +82-54-770-8501 \\ E-mail:jhseul@med.yu.ac.kr
}

This article was presented as a poster at the 67th Congress of the Korean Society of Plastic and Reconstructive Surgeons on November 19-21, 2009 in Seoul, Korea.

No potential conflict of interest relevant to this article was reported.

\section{INTRODUCTION}

Sweet's syndrome is characterized by clinical symptoms, pathologic findings which include fever, neutrophilia, tender erythematous skin lesions, and predominantly neutrophilic infiltration in the dermis [1]. Sweet's syndrome can be subdivided into three main forms: classical (or idiopathic), malignancy associated, and drug-induced Sweet's syndrome [2]. Recently, a "histiocytoid" pathologic variant of Sweet's syndrome has been described, and has been named histiocytoid Sweet's syndrome [3]. There are several disorders whose dermatologic symptoms can morphologically mimic those of Sweet's syndrome. We present a confusing case in which the patient initially appeared to have parotitis but turned out to have histiocytoid Sweet's syndrome.

\section{CASE}

A 57-year-old man reported swelling and tenderness on his right cheek for two months (Fig. 1). There was a history of upper respiratory tract infection two weeks earlier. We assumed that parotitis was caused by obstruction of the parotid duct, but outpatient treatment with oral antibiotics did not result in improvement. Furthermore, right sialography revealed no obstruction signs and no mass lesions (Fig. 2). During outpatient follow-up, erythematous papules and plaques started to appear on his hand and periocular regions (Fig. 3). Laboratory studies showed normal levels of neutrophils, except for an increased level of Creactive protein $(3.77 \mathrm{mg} / \mathrm{dL})$ and erythrocyte sedimentation rate $(95 \mathrm{~mm} / \mathrm{hr})$. We suspected autoimmune disease, and thus 
a skin punch biopsy was performed on the dorsum plaque of the right hand. Histopathologically, superficial perivenular histiocytic infiltration without vasculitis and nuclear debri admixed
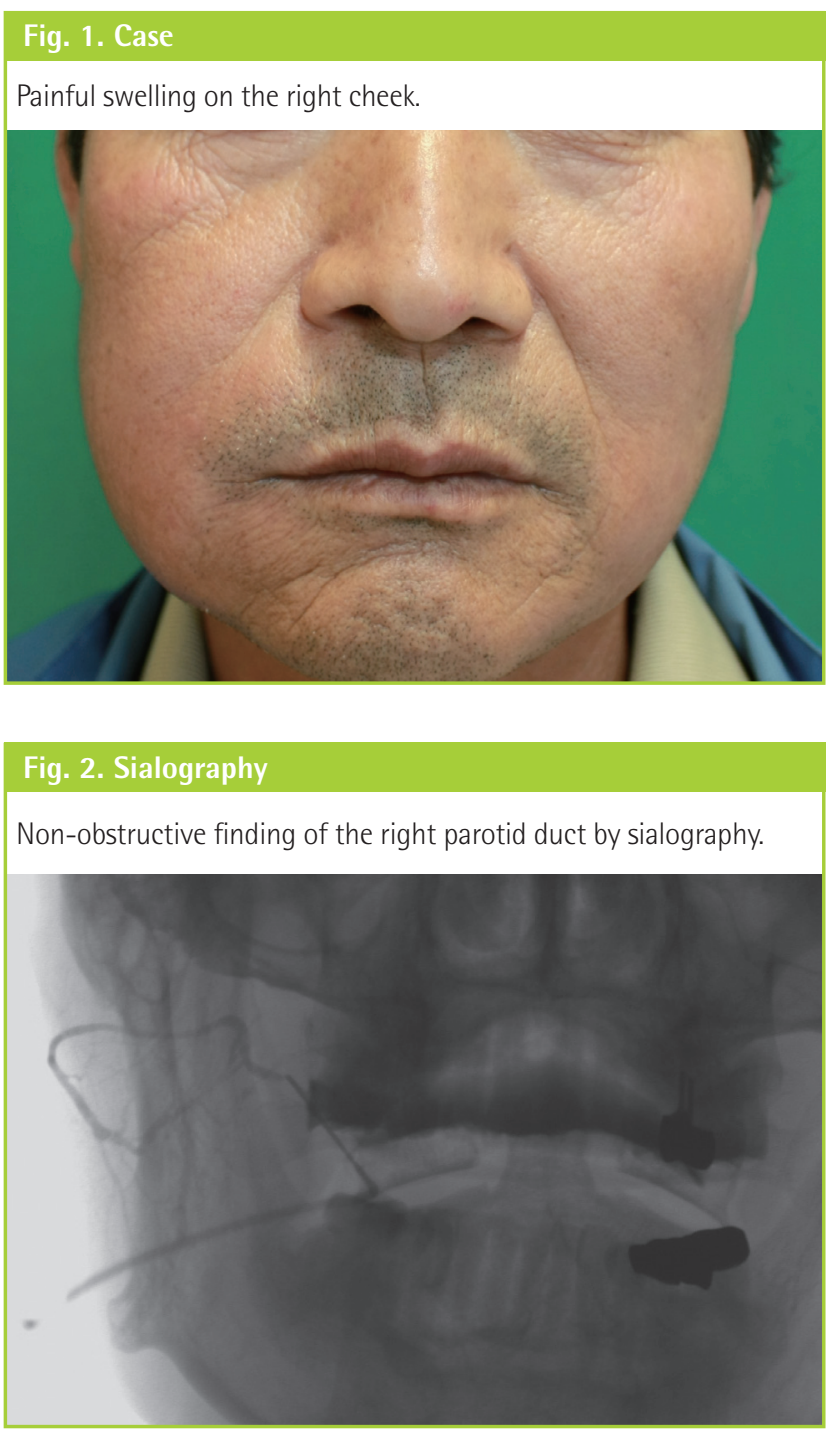

with papillary edema were seen in erythematous papules on the dorsum of the right hand (Fig. 4). After the histopathological and clinical diagnosis of Sweet's syndrome was made, systemic methylprednisolone was administered, starting with $32 \mathrm{mg}$ per day, for 2 weeks. The dose was tapered to $4 \mathrm{mg}$ over the course of the next 2 weeks. He responded with healing of the cutaneous lesions and of cheek swelling within a few days from the beginning of therapy.

\section{DISCUSSION}

Sweet's syndrome was originally described by Dr. Sweet [1] in 1964. It is characterized by fever, neutrophilia, tender erythematous skin lesions, and histopathologic evidence of a dense neutrophilic infiltration on the papillary dermis without vasculitis [1]. Sweet's syndrome has a worldwide distribution and no racial predilection. An annual incidence of 2.7 cases per $10^{6}$

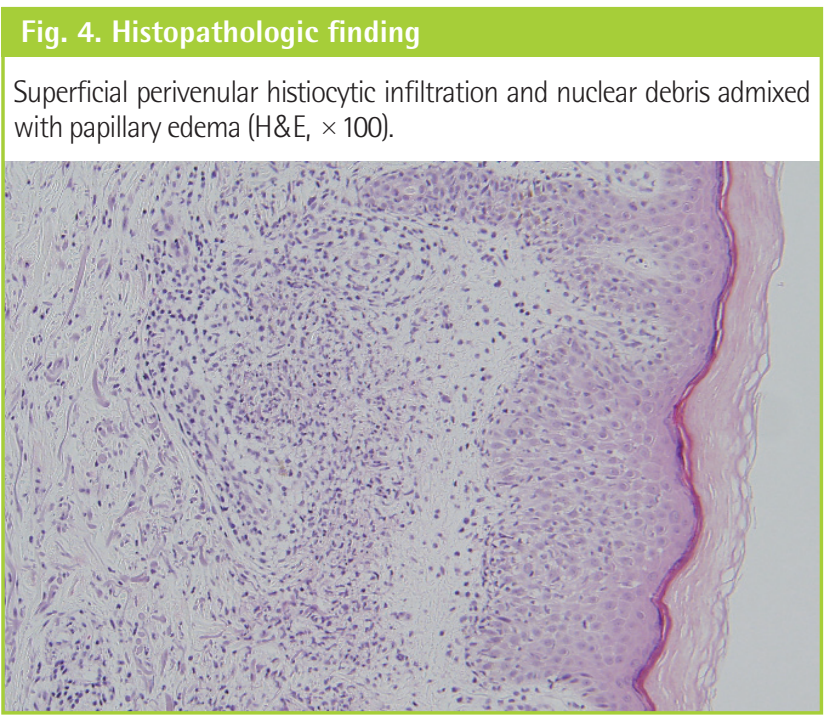

\section{Fig. 3. Postoperative follow-up}

(A) Erythematous macules on both periocular areas. (B) Erythematous papules and plaques on the dorsa of both hands.
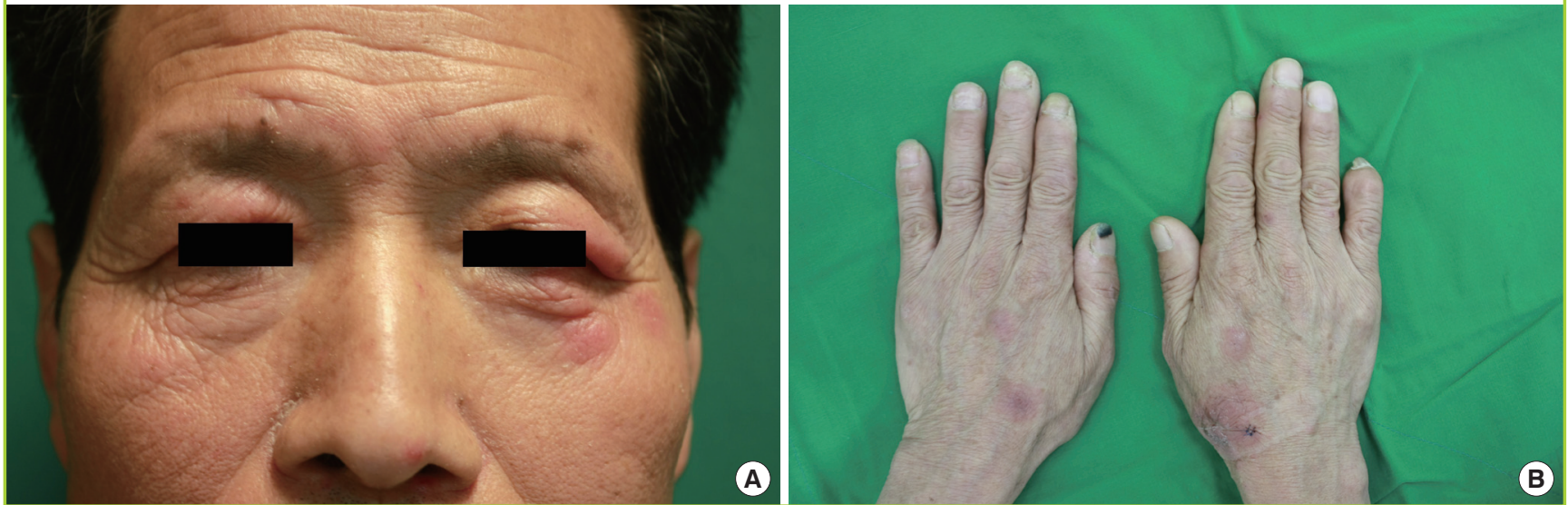
Table 1. Diagnostic criteria for classical Sweet's syndrome

\begin{tabular}{|l}
\hline \\
\hline Major criteria \\
Minor criteria \\
1. Abrupt onset of tender or painful erythematous plaques or nodules occasionally with vesicles, pustules or bullae \\
2. Predominantly neutrophilic infiltration in the dermis without leukocytoclastic vasculitis \\
1. Preceded by a nonspecific respiratory or gastrointestinal tract infection or vaccination or associated with: \\
1) Inflammation disease such as chronic autoimmune disorders, infections \\
2) Hemoproliferative disorders or solid malignant tumors \\
3) Pregnancy \\
2. Accompanied by periods of general malaise and fever ( $\left.>38^{\circ} \mathrm{C}\right)$ \\
3. Laboratory values during onset (three of four of these values necessary): \\
1) ESR $>20 \mathrm{~mm} / \mathrm{hr}$ \\
2) C-reactive protein positive \\
3) Segmented-nuclear neutrophils and stabs $>70 \%$ in peripheral blood smear \\
4) Leukocytosis $>8,000 / \mu \mathrm{L}$ \\
4. Excellent response to treatment with systemic corticosteroids or potassium iodide \\
\hline From von den Driesch P. J Am Acad Dermatol 1994;31:535-56, with permission from Elsevier [5]. \\
ESR, erythrocyte sedimentation rate.
\end{tabular}

population was estimated in Scotland [4]. The disease can occur in patients of any age. The female sex predominates, with a ratio of 3.7:1 [5]. Reported data about the incidence and prevalence of Sweet's syndrome in Korea does not exist.

The diagnostic criteria for Sweet's syndrome were first proposed by Su and Liu [6] in 1986. The criteria were modified by von den Driesch [5] in 1994. According to these criteria, for the diagnosis, two of the major criteria and two of the minor criteria should be fulfilled (Table 1). In our patient one major criterion, that abrupt onset of tender erythematous nodules and three minor criteria (associated upper respiratory infection, pathologic laboratory findings, and immediate response to systemic corticosteroids therapy) were fulfilled. The remaining major criterion is histopathologically dense infiltrate of mature neutrophils in the dermis. This case showed histiocytic infiltration with papillary edema instead of the typical neutrophil infiltration in the dermis. Therefore, we conclude that our patient had histiocytoid Sweet's syndrome, which was first described by Requena et al. [3] in 2005. Requena et al. [3] studied 42 cases of Sweet's syndrome, which were histopathologically characterized by an inflammatory infiltrate composed mostly of histiocytoid mononuclear cells. Based on the immunophenotype, they named this variant 'histiocytoid Sweet's syndrome'. According to the article, the apparent histiocytic mononuclear cells were in fact immature myeloid cells. Probably these lesions result from the release of immature myeloid cells from the bone marrow in the early acute stages of the disease, and these immature myeloid cells are replaced by more mature neutrophilic granulocytes in the later stages of the disease's evolution. The presence of the histiocytelike immature myeloid cells does not alter the biological behavior or prognosis of the disease. It is really a histological diagnosis. In general, the lesions follow a benign course, responding promptly to low doses of oral corticosteroids or nonsteroidal anti-inflammatory drugs.

Other conditions to be distinguished from Sweet's syndrome are erythema multiforme, Behcet's disease, drug eruption, periarteritis nodosa and infectious disorders such as bacterial sepsis, cellulitis, and lymphangitis [7]. Furthermore, Sweet's syndrome is an important differential diagnosis to postscald infections [8].

Systemic corticosteroids are the gold standard of therapy for Sweet's syndrome. They provide prompt relief of cutaneous and systemic symptoms. Systemic corticosteroid therapy begins with 0.5 to $1.5 \mathrm{mg} / \mathrm{kg}$ of body weight per day. Gradual reduction is recommended within 2 to 4 weeks [5].

In our patient, initially tender swelling was seen in the right cheek, such that parotitis was suspected. However, as additional skin lesions appeared, it turned out to be Sweet's syndrome. In conclusion, Sweet's syndrome should be considered in patients with therapy refractory unclear infiltrated nodules and tender swelling, especially in the face and associated with infection and fever.

\section{REFERENCES}

1. Sweet RD. An Acute febrile neutrophilic dermatosis. $\mathrm{Br} \mathrm{J}$ Dermatol 1964;76:349-56.

2. Cohen PR. Sweet's syndrome: a comprehensive review of an acute febrile neutrophilic dermatosis. Orphanet J Rare Dis 2007;2:34.

3. Requena L, Kutzner H, Palmedo G, et al. Histiocytoid Sweet syndrome: a dermal infiltration of immature neutrophilic granulocytes. Arch Dermatol 2005; 141:834-42.

4. Kemmett D, Hunter JA. Sweet's syndrome: a clinicopathologic review of twenty-nine cases. J Am Acad Dermatol 1990;23:503-7.

5. von den Driesch P. Sweet's syndrome (acute febrile neutro- 
philic dermatosis). J Am Acad Dermatol 1994;31:535-56.

6. Su WP, Liu HN. Diagnostic criteria for Sweet's syndrome. Cutis 1986;37:167-74.

7. Cohen PR, Kurzrock R. Sweet's syndrome: a neutrophilic dermatosis classically associated with acute onset and fever.
Clin Dermatol 2000;18:265-82.

8. Phua YS, Al-Ani SA, She RB, et al. Sweet's syndrome triggered by scalding: a case study and review of the literature. Burns 2010;36:e49-52. 